\title{
HIV knowledge, service utilization, and exposure to DREAMS among male partners of adolescent girls and young women in Malawi
}

Project SOAR

Follow this and additional works at: https://knowledgecommons.popcouncil.org/departments_sbsr-hiv

Part of the Public Health Commons

How does access to this work benefit you? Let us know!

\section{Recommended Citation}

Project SOAR. 2021. "HIV knowledge, service utilization, and exposure to DREAMS among male partners of adolescent girls and young women in Malawi," Project SOAR Results Brief. Washington, DC: Population Council. 


\section{HIV Knowledge, Service Utilization, and Exposure to DREAMS among Male Partners of Adolescent Girls and Young Women in Malawi}

In Malawi, HIV prevalence among adolescent girls and young women (AGYW), aged 15-24, is 2.3 times higher than among young men of the same age (3.4 percent vs. 1.5 percent). ${ }^{1}$ As in many countries, AGYW in Malawi are particularly vulnerable to HIV for a variety of reasons, including economic disadvantage and unequal power dynamics in sexual relationships with their male partners-many of whom are older and engage in high-risk behaviors. ${ }^{2}$ Additionally, compared to women, men are less likely to know their status, initiate HIV treatment, and are more likely to die of AIDS ${ }^{2,3}$-harming both themselves and their partners.

Project SOAR conducted research in 2019 among men in Malawi to learn more about their relationships with AGYW and how HIV services can better engage them.

Researchers surveyed 612 men ages 18 or older recruited at community hotspot venues and HIV service sites. Key inclusion criteria were being in an intimate or sexual relationship with an AGYW and residing in Zomba or Machinga districts.

This brief summarizes findings on men's knowledge of HIV, use of HIV services, stigma, and exposure to PEPFAR's DREAMS (Determined Resilient Empowered AIDS-free Mentored and Safe) program which took a multi-sectoral approach to reduce HIV risk among adolescent girls and young women and their male partners. ${ }^{4}$

\section{WHO WERE THE RESPONDENTS?}

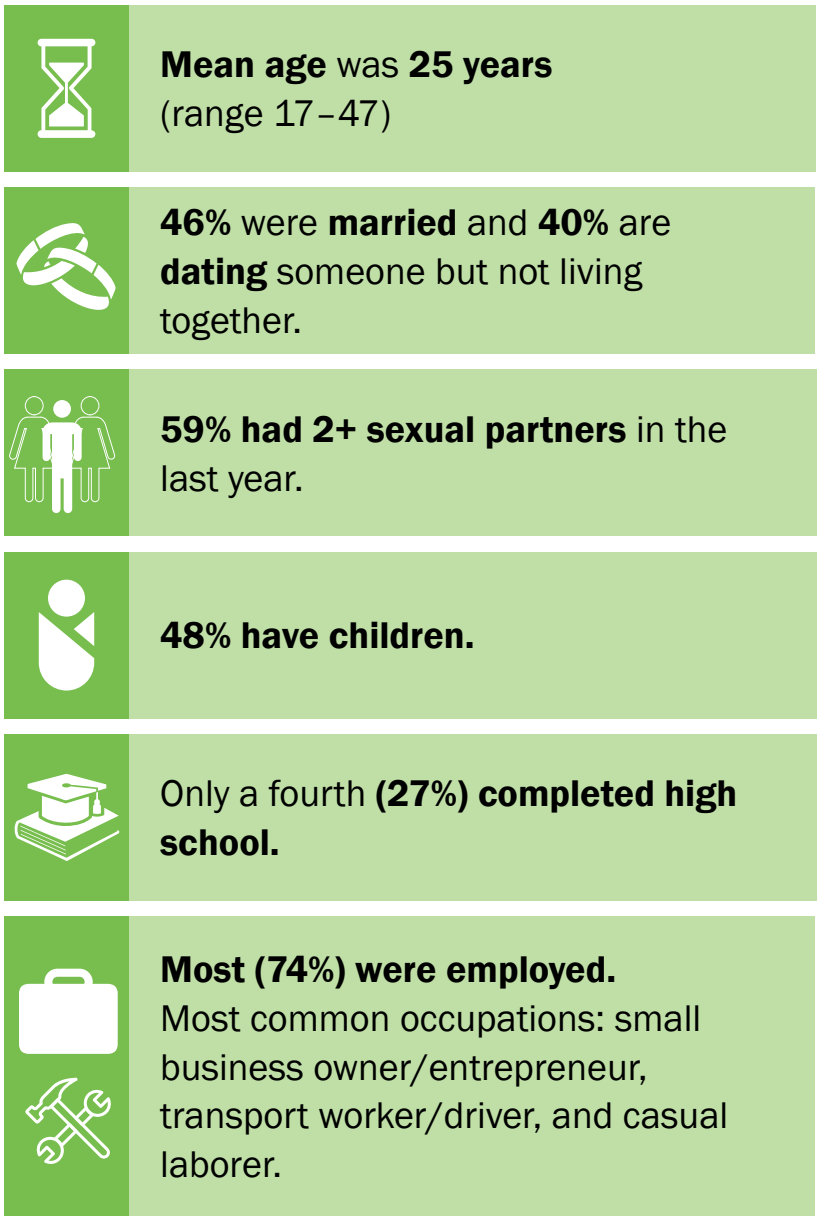

Interested in learning more about the male partners of AGYW? See the companion brief:

Characteristics and relationship dynamics of male partners of adolescent girls and young women in Malawi

Available at projsoar.org/our-activities/malawidreams 


\section{RESULTS}

\section{Comprehensive knowledge of HIV and condoms was low.}

Less than half (48 percent) answered all six questions about HIV correctly and barely a third (32 percent) correctly answered all five questions about condoms.

\section{Men had basic treatment literacy, but there were important gaps.}

The vast majority were aware of antiretroviral therapy (ART) (94 percent), knew that ART helps a person stay healthy (93 percent), and there are drugs a pregnant woman can take to prevent transmission to her baby (86 percent). But fewer men understood treatment as prevention ("ART reduces the risk of transmitting HIV to another person") and PrEP ("There are drugs an HIVnegative person can take to help prevent acquiring HIV") (Figure 1).

\section{Figure 1 HIV knowledge gaps}

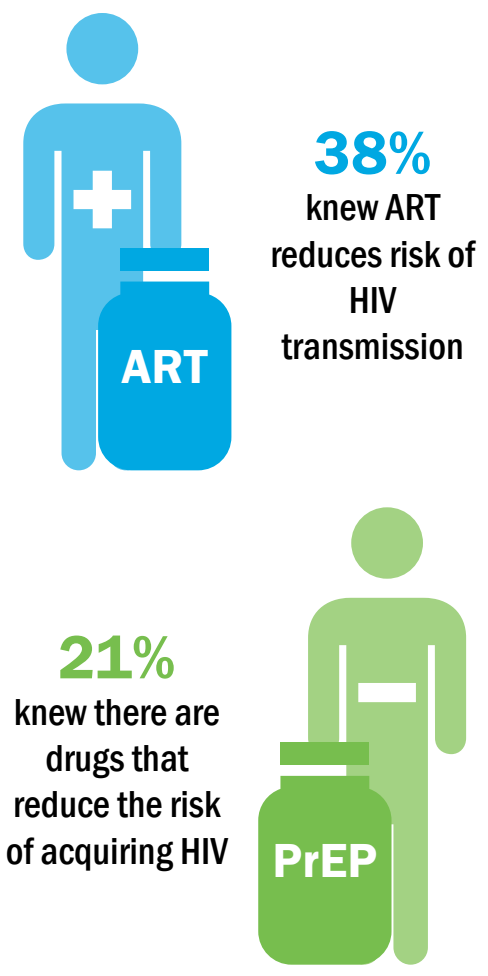

\section{HIV testing was very high among this population.}

A majority (96 percent) had ever tested and 84 percent had tested in the last 12 months. Repeat testing was very common-for only 3 percent the last test was the first time they had ever tested.

Men are using a variety of modalities to test other than a clinic/facility. Among those who tested in the last year, men learned their status through self-testing, a community venue, or at home (Figure 2). Another 7 percent underwent index testing.

\section{Figure 2 HIV testing modalities other than a clinic/facility in the past year

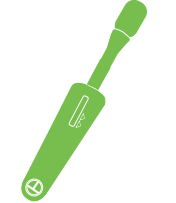 \\ $22 \%$ \\ self-testing
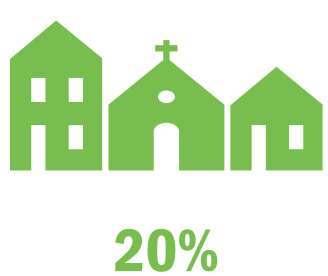 \\ community venue

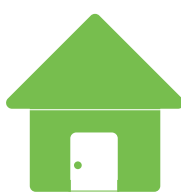 \\ $19 \%$ \\ home}

The main reason men cited for getting tested was wanting to know their status (69 percent). Fewer indicated they were tested as part of routine care (12 percent) or that a conversation or support from a spouse/partner triggered their desire to test ( 5 percent). Few ( 4 percent) indicated they had tested with or were accompanied by their spouse/partner.

\section{Many men anticipated a stigmatizing response if they disclosed that they tested positive for HIV.}

More than a third of men would be somewhat likely or very likely to:

- Experience a break-up of their marriage or relationship (36 percent).

- Experience physical abuse by their spouse/ partner (36 percent).

- Have difficulty finding sexual partners (41 percent).

- Have difficulty getting married (40 percent). 


\section{Men's practice of HIV preventive behaviors was uneven.}

The vast majority of men (88 percent) were circumcised and had been circumcised for traditional or religious reasons (rather than for HIV prevention). Among men who were circumcised as adolescents or young adults, about half reported receiving medical male circumcision, while the rest reported traditional circumcision. Among those not circumcised, most (62 percent) were considering undertaking the procedure. The average age of respondents considering circumcision was 26 years (age range 18-39 years).

Nearly three-fourths said they knew their partner's HIV status.

Health-seeking behavior was high among men who suspected they had a sexually transmitted infection (STI). Among the 13 percent who had experienced an STI symptom, 70 percent sought treatment.

But less than half used a condom at last sex with their current or most recent partner, which is low given that most men had multiple partners in the last year (Figure 3).
Figure 3 Practice of preventive behaviors
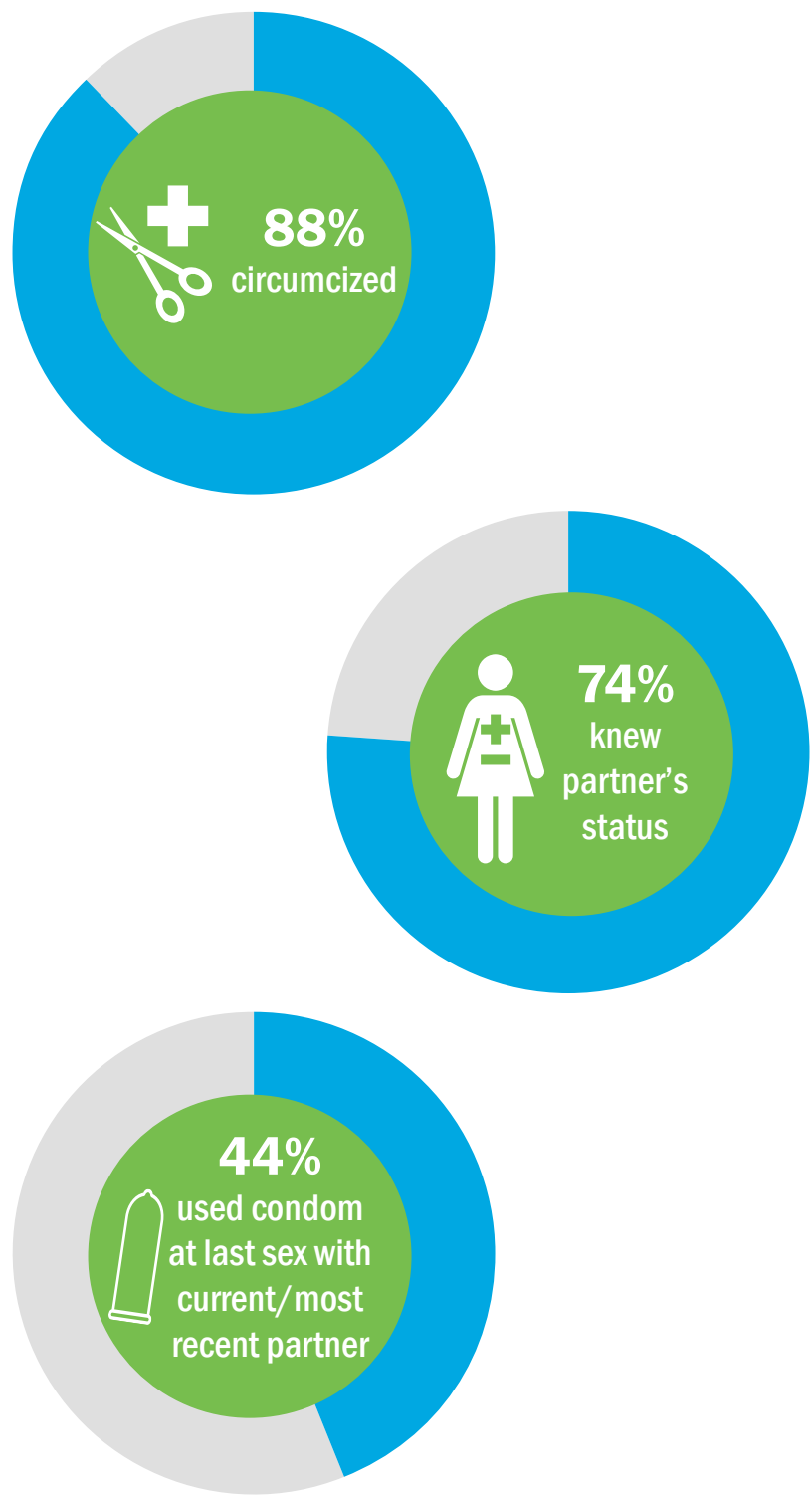

\section{Many men have heard of DREAMS and participated in an HIV-related event.}

Almost a half (43 percent) heard of DREAMS or Go Girls! Clubs. Of those, 26 percent had a female partner who participated in the program.

More than a third (34 percent) attended a community discussion group and/or one-on-one meeting in the last 12 months. All said that these events covered aspects of HIV prevention, care, or treatment. The vast majority reported that 
they also covered relationship issues, gender dynamics, or stigma (Figure 4).

\section{Figure 4 Topics discussed in community discussions/one-on-one meetings $(\mathbf{n}=211)$}
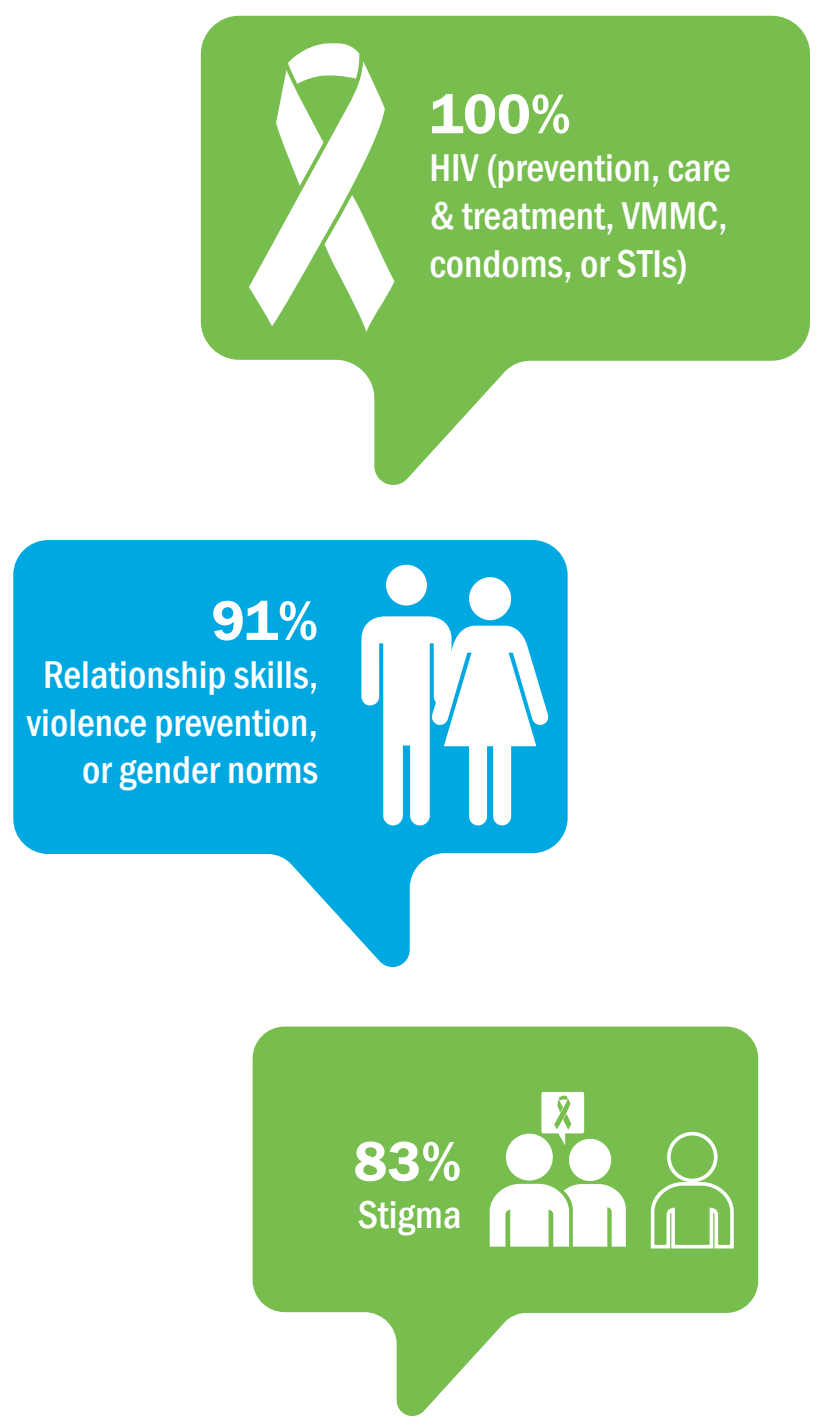

\section{REFERENCES}

1. Ministry of Health Malawi. 2018. "Malawi Population-Based HIV Impact Assessment (MPHIA) 2015-2016: final report." Lilongwe: Ministry of Health.

2. UNAIDS. 2016. "Prevention GAP report." Geneva: UNAIDS.

3. UNAIDS 2017. "Blind spot-reaching out to men and boys." Geneva: UNAIDS. https://www.unaids. org/sites/default/files/media_asset/blind_spot_ en.pdf

4. Saul, J et al. 2018. "The DREAMS core package of interventions: A comprehensive approach to preventing HIV among adolescent girls and young women," PLoS ONE 13(12): e0208167. doi: 10.1371/journal.pone.0208167

Suggested citation: Project SOAR. 2021. "HIV knowledge, service utilization, and exposure to DREAMS among male partners of adolescent girls and young women in Malawi," Project SOAR Results Brief. Washigton, DC: Population Council.
Project SOAR is a six-year+ (September 2014-January 2021) cooperative agreement funded by the United States President's Emergency Plan for AIDS Relief and the U. S. Agency for International Development (Agreement No. AID-OAA-A-14-00060). The contents of this brief are the sole responsibility of Project SOAR and Population Council and do not necessarily reflect the views of PEPFAR, USAID, or the United States Government.

Population Council leads the Project SOAR consortium in collaboration with Avenir Health, Elizabeth Glaser Pediatric AIDS Foundation, the Johns Hopkins University, Palladium, and The University of North Carolina at Chapel Hill.
Project SOAR/Population Council

4301 Connecticut Avenue, NW, Suite 280

Washington, DC 20008

Tel: +1 2022379400

e-mail: ProjectSOAR@popcouncil.org projsoar.org

(C)Population Council, January 2021 